\title{
Beyond 1/f: Multifractality in Human Heartbeat Dynamics
}

\author{
Plamen Ch. Ivanov ${ }^{1,2}$, Luís A. Nunes Amaral ${ }^{1,2}$, \\ Ary L. Goldberger ${ }^{2}$, Shlomo Havlin ${ }^{3}$, \\ Michael G. Rosenblum ${ }^{4}$, Zbigniew Struzik ${ }^{5}$, and H. Eugene Stanley ${ }^{1}$ \\ ${ }^{1}$ Center for Polymer Studies and Department of Physics, Boston University, Boston, MA 02215 \\ ${ }^{2}$ Harvard Medical School, Beth Israel Deaconess Medical Center, Boston, MA 02215 \\ ${ }^{3}$ Gonda Goldschmid Center and Department of Physics, Bar-Ilan University, Ramat Gan, Israel \\ ${ }^{4}$ Department of Physics, Potsdam University, D-14415 Potsdam, Germany \\ ${ }^{5}$ Centre for Math. and Comp. Science, Kruislaan 413, NL-1098 SJ Amsterdam, The \\ Netherlands
}

\begin{abstract}
Recent evidence suggests that physiological signals under healthy conditions may have a fractal temporal structure. We investigate the possibility that time series generated by certain physiological control systems may be members of a special class of complex processes, termed multifractal, which require a large number of exponents to characterize their scaling properties. We report on evidence for multifractality in a biological dynamical system - the healthy human heartbeat. Further, we show that the multifractal character and nonlinear properties of the healthy heart rate are encoded in the Fourier phases. We uncover a loss of multifractality for a life-threatening condition, congestive heart failure.
\end{abstract}

Biomedical signals are generated by complex self-regulating systems that process inputs with a broad range of characteristics [1,2]. Many physiological time series such as the one shown in Fig. 1a, are extremely inhomogeneous and nonstationary, fluctuating in an irregular and complex manner. The analysis of the fractal properties of such fluctuations has been restricted to second order linear characteristics such as the power spectrum and the two-point autocorrelation function. These analyses reveal that the fractal behavior of healthy, free-running physiological systems is often characterized by $1 / f$-like scaling of the power spectra [3-6].

Monofractal signals are homogeneous in the sense that they have the same scaling properties throughout the entire signal. Therefore monofractal signals can be indexed by a single global exponent--the Hurst exponent $H$ [12]. On the other hand, multifractal signals, can be decomposed into many subsets characterized by different local Hurst exponents $h$, which quantify the local singular behavior and thus relate to the local scaling of the time series. Thus multifractal signals require

CP511, Unsolved Problems of Noise and Fluctuations, edited by D. Abbott and L. B. Kish (C) 2000 American Institute of Physics 1-56396-826-6/00/ $\$ 17.00$ 


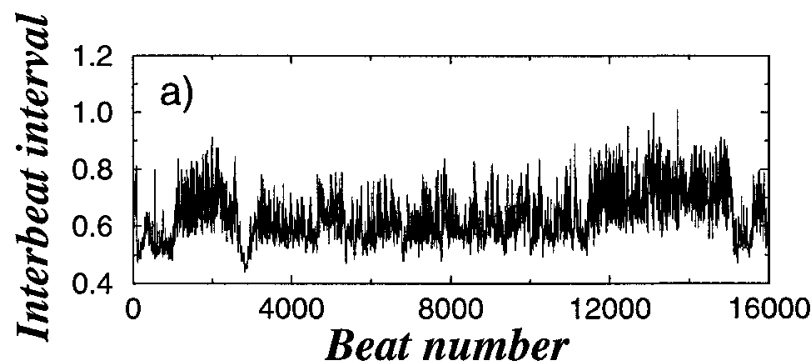

b)

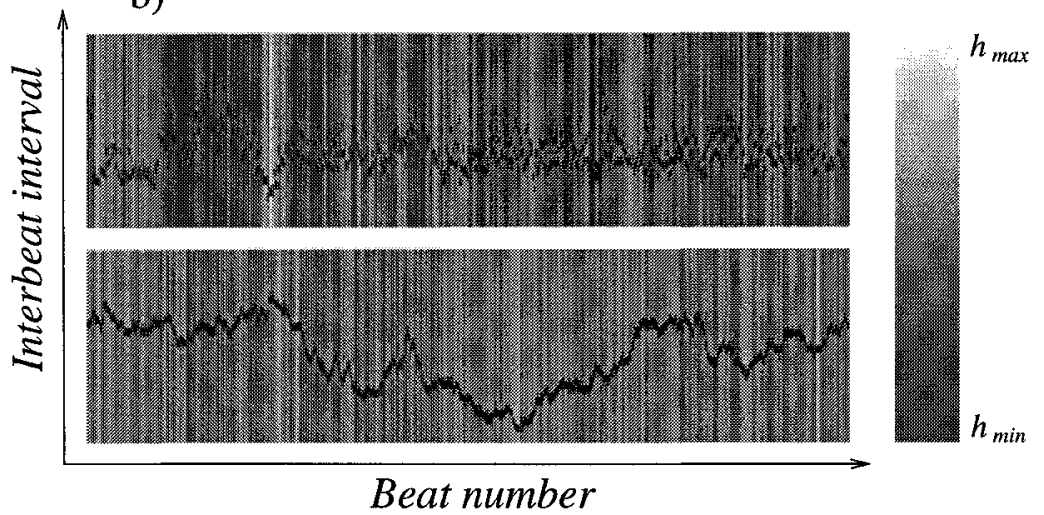

FIGURE 1. (a) Consecutive heartbeat intervals measured in seconds are plotted vs beat number from approximately 3 hours record of a representative healthy subject. The time series exhibits very irregular and nonstationary behavior. (b) The top panel displays in color the local Hurst exponents calculated for the same 3 hours record shown in (a). The heterogeneity of the healthy heartbeat is represented by the broad range of local Hurst exponents $h$ (colors) present and the complex temporal organization of the different exponents. The bottom panel displays in color the local Hurst exponents calculated for a monofractal signal - fractional Brownian motion with $H=0.6$. The homogeneity of the signal is represented by the nearly monochromatic appearance of the signal which indicates that the local Hurst exponent $h$ is the same throughout the signal and identical to the global Hurst exponent $H$. 
many exponents to fully characterize their scaling properties [7-9].

The statistical properties of the different subsets characterized by these different exponents $h$ can be quantified by the function $D(h)$, where $D\left(h_{o}\right)$ is the fractal dimension of the subset of the time series characterized by the local Hurst exponent $h_{o}[10,11,7-9]$. Thus, the multifractal approach for signals, a concept introduced in the context of multi-affine functions $[13,14]$, has the potential to describe a wide class of signals that are more complex then those characterized by a single fractal dimension (such as classical $1 / \mathrm{f}$ noise).

We test whether a large number of exponents is required to characterize heterogeneous heartbeat interval time series [Fig. 1] by undertaking multifractal analysis. The first problem is to extract the local value of $h$. To this end we use methods derived from wavelet theory [15]. The properties of the wavelet transform make wavelet methods attractive for the analysis of complex nonstationary time series such as one encounters in physiology [16]. In particular, wavelets can remove polynomial trends that could lead box-counting techniques to fail to quantify the local scaling of the signal [17]. Additionally, the time-frequency localization properties of the wavelets makes them particularly useful for the task of revealing the underlying hierarchy that governs the temporal distribution of the local Hurst exponents [18]. Hence, the wavelet transform enables a reliable multifractal analysis $[17,18]$. As the analyzing wavelet, we use derivatives of the Gaussian function, which allows us to estimate the singular behavior and the corresponding exponent $h$ at a given location in the time series. The higher the order $n$ of the derivative, the higher the order of the polynomial trends removed and the better the detection of the temporal structure of the local scaling exponents in the signal.

We evaluate the local exponent $h$ through the modulus of the maxima values of the wavelet transform at each point in the time series. We then estimate the scaling of the partition function $Z_{q}(a)$, which is defined as the sum of the $q^{\text {th }}$ powers of the local maxima of the modulus of the wavelet transform coefficients at scale $a$ [18]. For small scales, we expect

$$
Z_{q}(a) \sim a^{\tau(q)} .
$$

For certain values of $q$, the exponents $\tau(q)$ have familiar meanings. In particular, $\tau(2)$ is related to the scaling exponent of the Fourier power spectra, $S(f) \sim 1 / f^{\beta}$, as $\beta=2+\tau(2)$. For positive $q, Z_{q}(a)$ reflects the scaling of the large fluctuations and strong singularities, while for negative $q, Z_{q}(a)$ reflects the scaling of the small fluctuations and weak singularities $[7,8]$. Thus, the scaling exponents $\tau(q)$ can reveal different aspects of cardiac dynamics. The wavelet transform modulus maxima approach we use proves superior to the standard box-counting techniques and the structure factor method, which are limited only to positive moments $q$ [18].

Monofractal signals display a linear $\tau(q)$ spectrum, $\tau(q)=q H-1$, where $H$ is the global Hurst exponent. For multifractal signals, $\tau(q)$ is a nonlinear function: $\tau(q)=q h(q)-D(h)$, where $h(q) \equiv d \tau(q) / d q$ is not constant. The fractal dimension $D(h)$, introduced earlier, is related to $\tau(q)$ through a Legendre transform, 

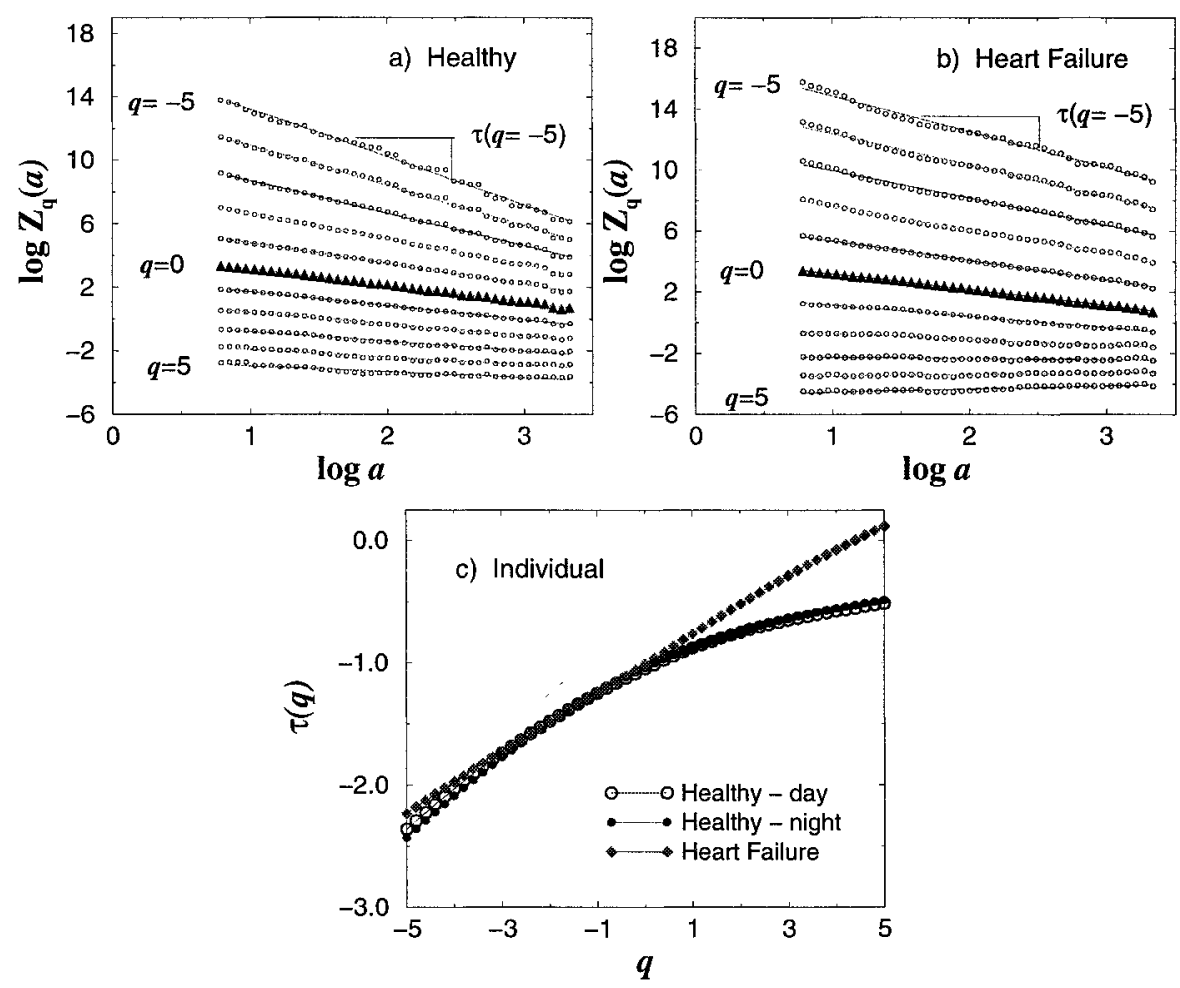

FIGURE 2. Heartbeat time series contain densely packed, non-isolated singularities which unavoidably affect each other in the time-frequency decomposition. Therefore, rather than evaluating the distribution of the inherently unstable local singularity exponents (as shown in color on Fig. 1), we estimate the scaling of an appropriately chosen global measure: the $q$ moments of the probability distribution of the maxima of the wavelet transform $Z_{q}(a)$ (as analyzing wavelet we use the 3rd derivative of the Gaussian function). Here we show the scaling of the partition function $Z_{q}(a)$ with scale $a$ obtained from daytime records consisting of $\approx 25,000$ beats for (a) a healthy subject and (b) a subject with congestive heart failure. We calculate $\tau(q)$ for moments $q=-5,4, \ldots, 0, \ldots, 5$ and scales $a=2 \times 1.15^{i}, i=0, \ldots, 41$. We display the calculated values of $Z_{q}(a)$ for scales $a>8$. The top curve corresponds to $q=-5$, the middle curve (shown heavy) to $q=0$ and the bottom curve to $q=5$. The exponents $\tau(q)$ are obtained from the slope of the curves in the region $16<a<700$, thus eliminating the influence of any residual small scale random noise due to ECG signal pre-processing as well as extreme, large scale fluctuations of the signal. (c) Multifractal spectrum $\tau(q)$ for individual records. A monofractal signal would correspond to a straight line for $\tau(q)$, while for a multifractal signal $\tau(q)$ is nonlinear. Note the clear differences between the curves for healthy and heart failure records. 

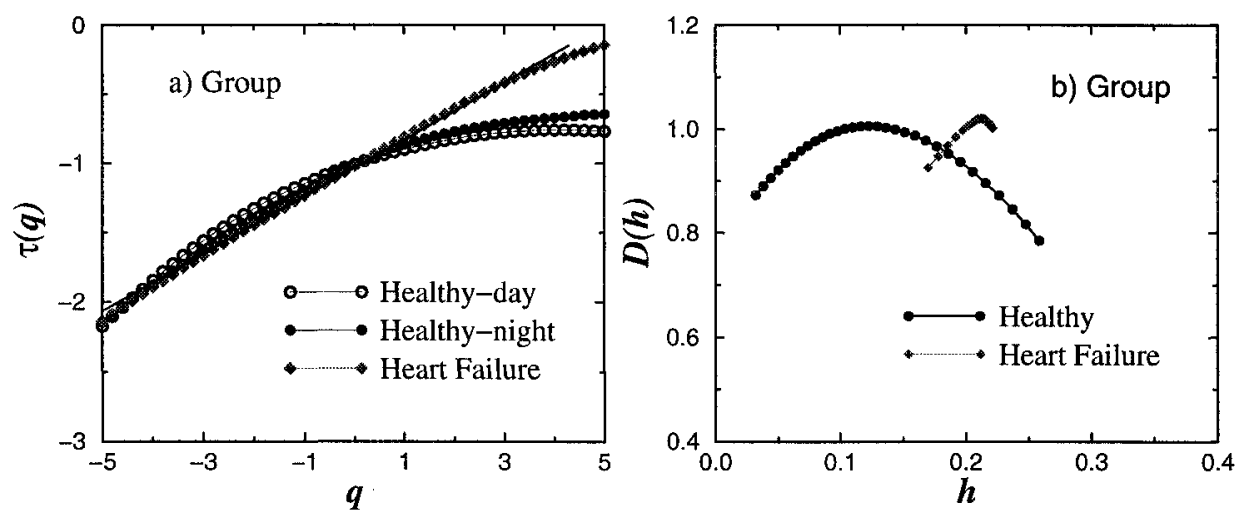

FIGURE 3. (a) Multifractal spectrum $\tau(q)$ of the group averages for daytime and nighttime records for 18 healthy subjects and for 12 patients with congestive heart failure. The results show multifractal behavior for the healthy group and distinct change in this behavior for the heart failure group. (b) Fractal dimensions $D(h)$ obtained through a Legendre transform from the group averaged $\tau(q)$ spectra of (a). The shape of $D(h)$ for the individual records and for the group average is broad, indicating multifractal behavior. On the other hand, $D(h)$ for the heart failure group is very narrow, indicating monofractality. The different form of $D(h)$ for the heart failure group may reflect perturbation of the cardiac neuroautonomic control mechanisms associated with this pathology.

$$
D(h)=q h-\tau(q) .
$$

We analyze both daytime (12:00 to 18:00) and nighttime (0:00 to $6: 00)$ heartbeat time series records of healthy subjects, and the daytime records of patients with congestive heart failure. These data were obtained by Holter monitoring. Our database includes 18 healthy subjects ( 13 female and 5 male, with ages between 20 and 50 , average 34.3 years), and 12 congestive heart failure subjects ( 3 female and 9 male, with ages between 22 and 71, average 60.8 years) in sinus rhythm [19]. For all subjects, we find that for a broad range of positive and negative $q$ the partition function $Z_{q}(a)$ scales as a power law [Figs. 2a,b].

For all healthy subjects, we find that $\tau(q)$ is a nonlinear function [Fig. 2c and Fig. 3a], which indicates that the heart rate of healthy humans is a multifractal signal. Figure $3 \mathrm{~b}$ shows that for healthy subjects, $D(h)$ has nonzero values for a broad range of local Hurst exponents $h$. The multifractality of healthy heartbeat dynamics cannot be explained by activity, as we analyze data from subjects during nocturnal hours. Furthermore, this multifractal behavior cannot be attributed to sleep-stage transitions, as we find multifractal features during daytime hours as well. The range of scaling exponents - $0<h<0.3$ - with nonzero fractal dimension $D(h)$, suggests that the fluctuations in the healthy heartbeat dynamics exhibit anti-correlated behavior $(h=1 / 2$ corresponds to uncorrelated behavior 
while $h>1 / 2$ corresponds to correlated behavior).

In contrast, we find that heart rate data from subjects with a pathological condition - congestive heart failure - show a clear loss of multifractality [Figs. 3a,b]. For the heart failure subjects, $\tau(q)$ is close to linear and $D(h)$ is non-zero only over a very narrow range of exponents $h$ indicating monofractal behaviour [Fig. 3].

Our results show that, for healthy subjects, local Hurst exponents in the range $0.07<h<0.17$ are associated with fractal dimensions close to one. This means that the subsets characterized by these local exponents are statistically dominant. On the other hand, for the heart failure subjects, we find that the statistically dominant exponents are confined to a narrow range of local Hurst exponents centered at $h \approx 0.22$. These results suggest that for heart failure the fluctuations are less anti-correlated than for healthy dynamics since the dominant scaling exponents $h$ are closer to $1 / 2$.

The multifractality of heart beat time series also enables us to quantify the greater complexity of the healthy dynamics compared to pathological conditions. Power spectrum analysis defines the complexity of heart beat dynamics through its scale-free behavior, identifying a single scaling exponent as an index of healthy or pathologic behavior. Hence, the power spectrum is not able to quantify the greater level of complexity of the healthy dynamics, reflected in the heterogeneity of the signal. On the other hand, the multifractal analysis reveals this new level of complexity by the broad range of exponents necessary to characterize the healthy dynamics. Moreover, the change in shape of the $D(h)$ curve for the heart failure group may provide insights into the alteration of the cardiac control mechanisms due to this pathology.

To further study the complexity of the healthy dynamics, we perform two tests with surrogate time series. First, we generate a surrogate time series by shuffling the interbeat interval increments of a record from a healthy subject. The new signal preserves the distribution of interbeat interval increments but destroys the long-range correlations among them. Hence, the signal is a simple random walk, which is characterized by a single Hurst exponent $H=1 / 2$ and exhibits monofractal behavior [Fig. 4a]. Second, we generate a surrogate time series by performing a Fourier transform on a record from a healthy subject, preserving the amplitudes of the Fourier transform but randomizing the phases, and then performing an inverse Fourier transform. This procedure eliminates nonlinearities, preserving only the linear features of the original time series. The new surrogate signal has the same $1 / f$ behavior in the power spectrum as the original heart beat time series; however it exhibits monofractal behavior [Fig. 4a]. We repeat this test on a record of a heart failure subject. In this case, we find a smaller change in the multifractal spectrum [Fig. 4b]. The results suggest that the healthy heartbeat time series contains important phase correlations cancelled in the surrogate signal by the randomization of the Fourier phases, and that these correlations are weaker in heart failure subjects. Furthermore, the tests indicate that the observed multifractality is related to nonlinear features of the healthy heartbeat dynamics. A number of recent studies have tested for nonlinear and deterministic properties in recordings of interbeat 

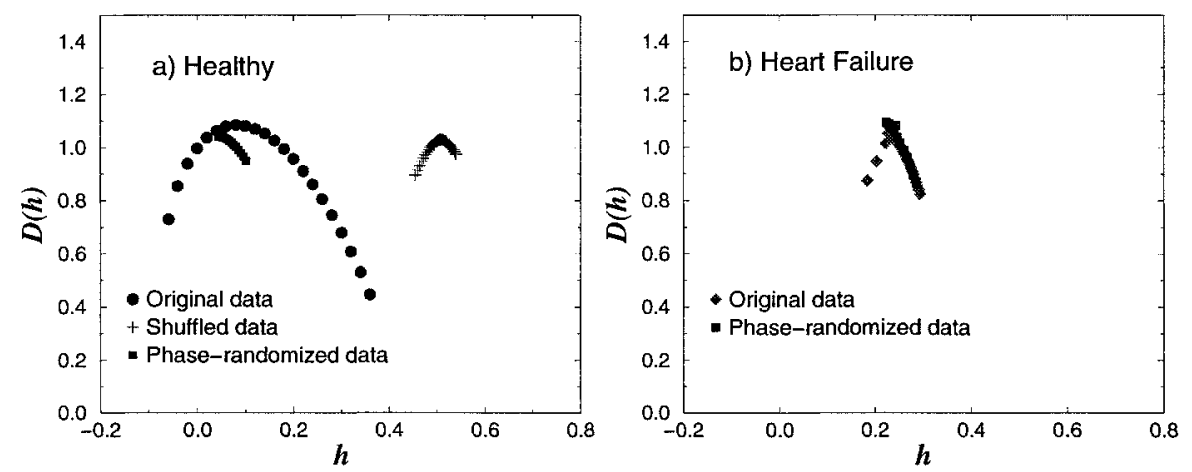

FIGURE 4. (a) The fractal dimensions $D(h)$ for a 6 h daytime record of a healthy subject. After reshuffling and integrating the increments in this interbeat interval time series, so that all correlations are lost but the distribution is preserved, we obtain monofractal behavior - a very narrow point-like spectrum centered at $h \equiv H=1 / 2$. Such behavior corresponds to a simple random walk. A different test, in which the $1 / f$-scaling of the heart beat signal is preserved but the Fourier phases are randomized (i.e., nonlinearities are eliminated) leads again to a monofractal spectrum centered at $h \approx 0.07$, since the linear correlations were preserved. These tests indicate that the observed multifractality is related to nonlinear features of the healthy heart beat dynamics rather than to the ordering or the distribution of the interbeat intervals in the time series. (b) The fractal dimensions $D(h)$ for a $6 \mathrm{~h}$ daytime record of a heart failure subject. The narrow multifractal spectrum indicates loss of multifractal complexity and reduction of nonlinearities with pathology.

intervals [20-23]. Our results are the first to demonstrate an explicit relation between the nonlinear features (represented by the Fourier phase interactions) and the multifractality of healthy cardiac dynamics [Fig. 4]. The exact nature of this relation, however, remains an open question.

From a physiological perspective, the detection of robust multifractal scaling in the heart rate dynamics is of interest because our findings raise the intriguing possibility that the control mechanisms regulating the heartbeat interact as part of a coupled cascade of feedback loops in a system operating far from equilibrium $[24,25]$. Furthermore, the present results indicate that the healthy heartbeat is even more complex than previously suspected, posing a challenge to ongoing efforts to develop realistic models of the control of heart rate and other processes under neuroautonomic regulation.

\section{REFERENCES}

1. Shlesinger, M. F. Ann. NY Acad. Sci. 504, 214-228, (1987).

2. Malik, M. and Camm, A. J. eds. Heart Rate Variability (Futura, Armonk NY, 1995). 
3. Akselrod, S. et al. Science 213, 220-222 (1981).

4. Kobayashi, M. and Musha, T. IEEE Trans. Biomed. Eng. 29, 456-457 (1982).

5. Hausdorff, J.M. et al. J. Appl. Physiol. 80, 1448-1457 (1996).

6. Peng, C.-K. et al. Chaos 5, 82 (1995).

7. Vicsek, T. Fractal Growth Phenomena, 2nd ed. (World Scientific, Singapore, 1993).

8. Takayasu, H. Fractals in the Physical Sciences (Manchester University Press, Manchester, UK, 1997).

9. Stanley, H. E. in Fractals and Disordered Systems, Second Edition, eds. A. Bunde, S. Havlin (Springer-Verlag, Berlin, 1996), pp. 1-68.

10. Dewey, T.G. Fractals in Molecular Biophysics (Oxford University Press, Oxford, 1997).

11. Stanley, H.E. and Meakin, P. Nature, 335, 405-409 (1988).

12. Hurst, H. E. Trans. Am. Soc. Civ. Eng. 116, 770-808 (1951).

13. Vicsek, T. \& Barabási, A.L. J. Phys. A: Math. Gen., 24, L845-L851 (1991).

14. Barabasi A.-L. and Stanley, H.E. Fractal Concepts in Surface Growth (Cambridge University Press, Cambridge, 1995), Chapter 24.

15. Daubechies, I. Ten Lectures on Wavelets (S.I.A.M., Philadelphia, 1992).

16. Ivanov, P.Ch. et al. Nature 383, 323-327 (1996).

17. Muzy, J.F., Bacry, E. and Arneodo, A. Phys. Rev. Lett. 67, 3515-3518 (1991).

18. Muzy, J.F., Bacry, E. and Arneodo, A. Int. J. Bifurc. Chaos. 4, 245-302 (1994).

19. Heart Failure Database (Beth Israel Deaconess Medical Center, Boston, MA). The database includes 18 healthy subjects $(13$ female and 5 male, with ages between 20 and 50, average 34.3 years), and 12 congestive heart failure subjects ( 3 female and 9 male, with ages between 22 and 71 , average 60.8 years) in sinus rhythm.

20. Lefebvre, J. et al. Chaos 3, 267-276 (1993).

21. Yamamoto, Y. et al. Biol. Cybern. 69, 205-212 (1993).

22. Kanters, J.K., Holstein-Rathlou, N.H. and Agner, E. J. Cardiovasc. Electrophysiol. 5, 128-137 (1994).

23. Sugihara, G. et al. Proc. Natl. Acad. Sci. USA 93, 2608-2613 (1996).

24. Meneveau, C. and Sreenivasan, K.R. Phys. Rev. Lett. 59, 1424-1427 (1987).

25. Ivanov, P.Ch. et al. Europhys. Lett. 43, 363-368 (1998). 